(MVD). Liver ultrasound was used to diagnose and grade the NAFLD (grade 1-3). NAFLD fibrosis score (NFS) was calculated, and patients were stratified to low, indeterminate, and high probability for advanced liver fibrosis.

Results Total of 85 patients with median age of 40 years (IQR 35-43) and predominated by males (84.7\%). Thirtythree (38.9\%) had ST elevation myocardial infarction (STEMI), $32(37.6 \%)$ had Non-STEMI and 20 (23.5\%) had unstable angina. MVD was demonstrated in $36.5 \%$, SVD in $24.7 \%$, mild CAD in $31.8 \%$, and no apparent CAD in $7.1 \%$ of the patients. Median Syntax score was 16 (IQR 9.0-22.3). NAFLD was diagnosed in all, $85(100 \%)$ patients, with 13 (15.2\%), $36(42.4 \%)$ and $36(42.2 \%)$ patients had grade 1, 2 and 3 liver steatosis respectively. NFS detected low advanced fibrosis probability in $60(70.6 \%)$ patients, indeterminate probability in $24(28.2 \%)$ patients and high probability in only 1 (1.2\%) patient. No significant correlation between grades of NAFLD with ACS subtypes $(p=0.72)$, severity of CAD $(\mathrm{p}=0.882)$ and SYNTAX score $(\mathrm{p}=0.982)$. No significant association between NFS and ACS subtypes $(p=0.232)$, severity of CAD $(p=0.445)$ and SYNTAX score $(p=0.624, r=0.07)$.

Conclusions NAFLD is highly prevalent in young patients presented with ACS, and it should be routinely screened in our clinical practice. However, in a small cohort, we observed no significant correlation between severity of NAFLD and severity of CAD among young ACS patients.

\section{IDDF2018-ABS-0235 REAPPRAISAL OF THE ACCURACY OF DIAGNOSTIC CRITERIA FOR SPONTANEOUS BACTERIAL PERITONITIS IN CIRRHOTIC PATIENTS WITH OR WITHOUT HEPATOCELLULAR CARCINOMA: A PRELIMINARY RESULT}

${ }^{1} \mathrm{Ha} \| \mathrm{Kim}$ * ${ }^{1}$ Ju Hyun Shim, ${ }^{2}$ Jihyun An, ${ }^{1}$ Sangyoung Yi, ${ }^{1}$ Jonggi Choi, ${ }^{1}$ Gwang Hyeon Choi, ${ }^{1}$ Danbi Lee, ${ }^{1}$ Kang Mo Kim, ${ }^{1}$ Young-Suk Lim, ${ }^{1}$ Han Chu Lee, ${ }^{1}$ Young-Hwa Chung, ${ }^{1}$ Yung Sang Lee. ${ }^{1}$ Asan medical center, Korea, South; ${ }^{2}$ Korea Advanced Institute of Science and Technology, Korea, South

\subsection{6/gutjnl-2018-IDDFabstracts.236}

Background Since 1976, the laboratory diagnosis of spontaneous bacterial peritonitis (SBP) has been established by asceticfluid (AF) polymorphonuclear-leucocyte (PMN) count $\geq 250$ / $\mathrm{mm}^{3}$ with or without the AF culture result in cirrhotic patients. We aimed to reevaluate whether the current cutoff count of PMN would be still optimal to diagnose SBP in cirrhotics having a hepatocellular carcinoma (HCC) or not.

Methods This preliminary study included 136 consecutive patients having cirrhosis with $(n=60)$ or without HCC $(n=76)$ and a positive AF culture at the first exploratory paracentesis between 2007-2016 as the SBP group; and 202 cirrhotics with neither bacterascites on the first AF specimen nor any typical symptoms suggestive of peritoneal infection as the nonSBP control group. Among the SBP group, none had a clinical suspicion of secondary peritonitis and malignant ascites.

Results Fever (28\%) and E. coli (22\%) were the most common symptom and isolated pathogen, respectively in the SBP group. Ascitic white-blood-cell and PMN counts, and lactatedehydrogenase were significantly higher in the SBP group, whereas glucose and serum-ascites-albumin-gradient were higher in the control group ( $\mathrm{Ps}<0.05$ ). Total-protein and $\mathrm{pH}$ in the AF were similar in both groups. For the entire SBP patients, receiver-operating-characteristic analysis to determine the diagnostic performance of ascetic-PMN $\geq 250 / \mathrm{mm}^{3}$ showed a sensitivity of $87 \%$ and a specificity of $95 \%$, comparable between the LC and HCC subsets. There were no differences in both AF results and symptoms between SBP patients with cirrhosis and HCC. The 90th-percentile limits for the asciticPMN counts were 246 and $466 / \mathrm{mm}^{3}$, respectively for the LC and HCC subsets with SBP.

Conclusions Based on our results, update or revision of the old threshold count for ascetic-PMN may be taken into consideration to be timely treated with antibiotics in not only HCC patients but cirrhotics who have established or suspected SBP, leading to prevention of undertreatment of serious infection and overtreatment of sterile ascites. Our further study of the extended cohort is ongoing.

\section{IDDF2018-ABS-0247 PORTAL CAVERNOMA IN A NON-CIRRHOTIC YOUNG FEMALE: A CASE REPORT}

Millette Castro. University of the East Ramon Magsaysay Memorial Medical Center, Philippines

\subsection{6/gutjnl-2018-IDDFabstracts.237}

Background Cavernous transformation of the portal vein is a rare entity and usually is a sequela of EHPV.

We report a case of a 21 year old Filipino female presented with a history of intermittent hematemesis which started when she was 1 year old. Pre-natal and birth history was unremarkable. She had recurrent episodes of upper GI bleeding even at a young age. No other stigmata of liver disease were noted. Endoscopic band ligation bleeding varices was done. Liver function tests, Hepatitis B and C screening were negative. Abdominal MRI and CT scan revealed portal cavernoma and absence of cirrhosis.

Methods We report a case of a young female adult in whom the diagnosis of CTPV was made after extensive workup for the case of her recurrent upper GI bleeding and signs of portal hypertension in the absence of cirrhosis and other stigmata of chronic liver diseases.

Results Complete blood count revealed bicytopenia and adequate platelets with microcytic, hypochromic anaemia. PBS revealed Microcytic cells with hypochromicity, anisocytosis and poikilocytosis. Liver function tests, Hepatitis B and C screening were negative. Fibroscan of the liver revealed intermediate fibrosis. Colour flow Doppler ultrasound of the abdomen is compatible with portal hypertension of the portal vein and splenomegaly. MRI of the whole abdomen revealed portal hypertension with marked splenomegaly, cavernous transformation of the portal vein and collateral vessel formation. There's also focal biliary ectasia and cholecystolithiasis. Repeat gastroscopy revealed 3 esophageal varices.

Conclusions In conclusion, NCPH is the most common cause of portal hypertension next to cirrhosis. Two disease entities in $\mathrm{NCPH}$, namely $\mathrm{NCPF} / \mathrm{IPH}$ and $\mathrm{EHPVO}$ are distinct diseases, presenting with features of PHT - variceal bleed, splenomegaly and near normal liver functions. Likely pathogenesis is early age, portal inflammation/infection in a prothrombotic individual although we still have to work up this patient for thrombotic risk. The diagnosis needs exclusion of cirrhosis in $\mathrm{NCPF} / \mathrm{IPH}$ and presence of portal cavernoma in EHPVO. Slow hepatic dysfunction due to parenchymal extinction and portal biliopathy is a late event in EHPVO. Effective management of PHT and its complications results in excellent 5 and 10 years survival. 